\title{
CONSTRAINTS ON CORONAL MASS EJECTION EVOLUTION FROM IN SITU OBSERVATIONS OF IONIC CHARGE STATES
}

\author{
Jacob R. Gruesbeck ${ }^{1}$, Susan T. Lepri ${ }^{1}$, Thomas H. Zurbuchen ${ }^{1}$, and Spiro K. Antiochos ${ }^{2}$ \\ ${ }^{1}$ Department of Atmospheric, Oceanic, and Space Science, University of Michigan, Ann Arbor, MI 48109, USA; jagruesb@umich.edu \\ 2 NASA Goddard Space Flight Center, Greenbelt, MD 20771, USA \\ Received 2010 October 19; accepted 2011 January 25; published 2011 March 10
}

\begin{abstract}
We present a novel procedure for deriving the physical properties of coronal mass ejections (CMEs) in the corona. Our methodology uses in situ measurements of ionic charge states of $\mathrm{C}, \mathrm{O}, \mathrm{Si}$, and $\mathrm{Fe}$ in the heliosphere and interprets them in the context of a model for the early evolution of interplanetary CME (ICME) plasma, between 2 and $5 R_{\odot}$. We find that the data are best fit by an evolution that consists of an initial heating of the plasma, followed by an expansion that ultimately results in cooling. The heating profile is consistent with a compression of coronal plasma due to flare reconnection jets and an expansion cooling due to the ejection, as expected from the standard CME/flare model. The observed frozen-in ionic charge states reflect this time history and, therefore, provide important constraints for the heating and expansion timescales, as well as the maximum temperature the CME plasma is heated to during its eruption. Furthermore, our analysis places severe limits on the possible density of CME plasma in the corona. We discuss the implications of our results for CME models and for future analysis of ICME plasma composition.
\end{abstract}

Key words: atomic processes - plasmas - solar wind - Sun: coronal mass ejections (CMEs)

Online-only material: color figures

\section{INTRODUCTION}

Coronal mass ejections (CMEs) are an important source of plasma and magnetic field in the heliosphere and the drivers of space weather events near Earth. They are characterized by the rapid and explosive release of large quantities of coronal material entrapped in its magnetic field. The rate of occurrence of CMEs is directly tied to the solar cycle (Gopalswamy et al. 2004) making them an important consequence of solar activity. Ejections from the Sun are more prevalent during solar maximum conditions and occur nearly 10 times less frequently (Gopalswamy et al. 2004) in the deepest of the solar minimum.

CMEs are observed remotely in many wavelengths including white-light observations, by the Large Angle Spectroscopic Coronagraph (LASCO; Brueckner et al. 1995), for example. Indeed, many CMEs are observed to be closely associated with enhanced plasma heating and energization, often relating to flare events during and immediately following the eruption (Harrison 1995; Vršnak et al. 2005). Extreme ultraviolet and soft $\mathrm{X}$-ray emissions are most often used to analyze such heating (Krucker et al. 2010). These energization events can release energetic particles which are observed in remote observations of soft X-ray (Dauphin et al. 2006), hard X-ray (Temmer et al. 2010; Goff et al. 2005), and gamma-ray (Lin et al. 2003). Energetic particles can further interact with plasma near the Sun, in particular near the chromosphere, and lead to chromospheric evaporation events. We can directly observe the heliospheric manifestations of the erupted plasma, the so-called interplanetary CMEs (ICMEs), which are characterized by specific plasma characteristics (Zurbuchen \& Richardson 2006).

ICMEs are usually associated with magnetically dominated plasma, which appear to be approximately in a Taylor state with smooth and near force-free magnetic fields (Burlaga et al. 1981). ICMEs observed near $1 \mathrm{AU}$ often have bulk speeds that exceed that of the surrounding solar wind and therefore are associated with interaction signatures, such as compressions or shocks (Zurbuchen \& Richardson 2006 and references therein). Additionally, there are important expansion signatures, such as steadily decreasing velocity profiles, and depressed proton temperatures and densities (Gosling et al. 1973; Richardson \& Cane 1995). Unusual charge state of heavy ions in the ICME plasma is another important signature (Bame et al. 1979; Henke et al. 1998; Lepri et al. 2001; Lepri \& Zurbuchen 2004; Rodriguez et al. 2004; Zurbuchen et al. 2003). In fact, these compositional signatures were recently shown to be more prevalent than initially assumed, making them a powerful tool in ICME identification (Richardson \& Cane 2004, 2010).

Figure 1 shows the plasma, magnetic field, and compositional signatures of an example ICME as observed with the Advanced Composition Explorer (ACE; Stone et al. 1998). The steadily decreasing velocity profile, the low density, and depressed temperatures are all signatures of an expanding magnetic cloud. The ICME is magnetically dominated and has the key characteristics of a flux-rope configuration.

Parts G-K of Figure 1 show measurements of the ionic distribution of $\mathrm{C}, \mathrm{O}, \mathrm{Si}$, and $\mathrm{Fe}$. Unusually high charge states in $\mathrm{C}$, $\mathrm{O}, \mathrm{Si}$, and $\mathrm{Fe}$ are the most important compositional signatures of this ICME, and many others, indicative of enhanced collisional ionization near the Sun. In fact, it has been reported that $50 \%$ of all ICMEs exhibit elevated charge states in Fe (Lepri et al. 2001) and generally exhibit elevated states in the other species as well. The oxygen charge distribution, which usually peaks at $\mathrm{O}^{6+}$ in the solar wind, peaks at $\mathrm{O}^{7+}$, which is also observed in the $\mathrm{O}^{7+} / \mathrm{O}^{6+}$ ratio, and has important contributions of $\mathrm{O}^{8+}$. Similarly, carbon charge distributions in ICMEs are observed to be strongly dominated by $\mathrm{C}^{6+}$, the fully ionized state. The most unusual ionic charge state related to ICME plasma is the Ne-like charge state of $\mathrm{Fe}(q=16)$ clearly visible in part $\mathrm{H}$ of Figure 1 (Lepri et al. 2001). Similarly, as we will discuss later, silicon tends to be observed preferentially with an $\mathrm{He}$-like charge state, ionized to $\mathrm{Si}^{12+}$, as shown in part I of Figure 1. 


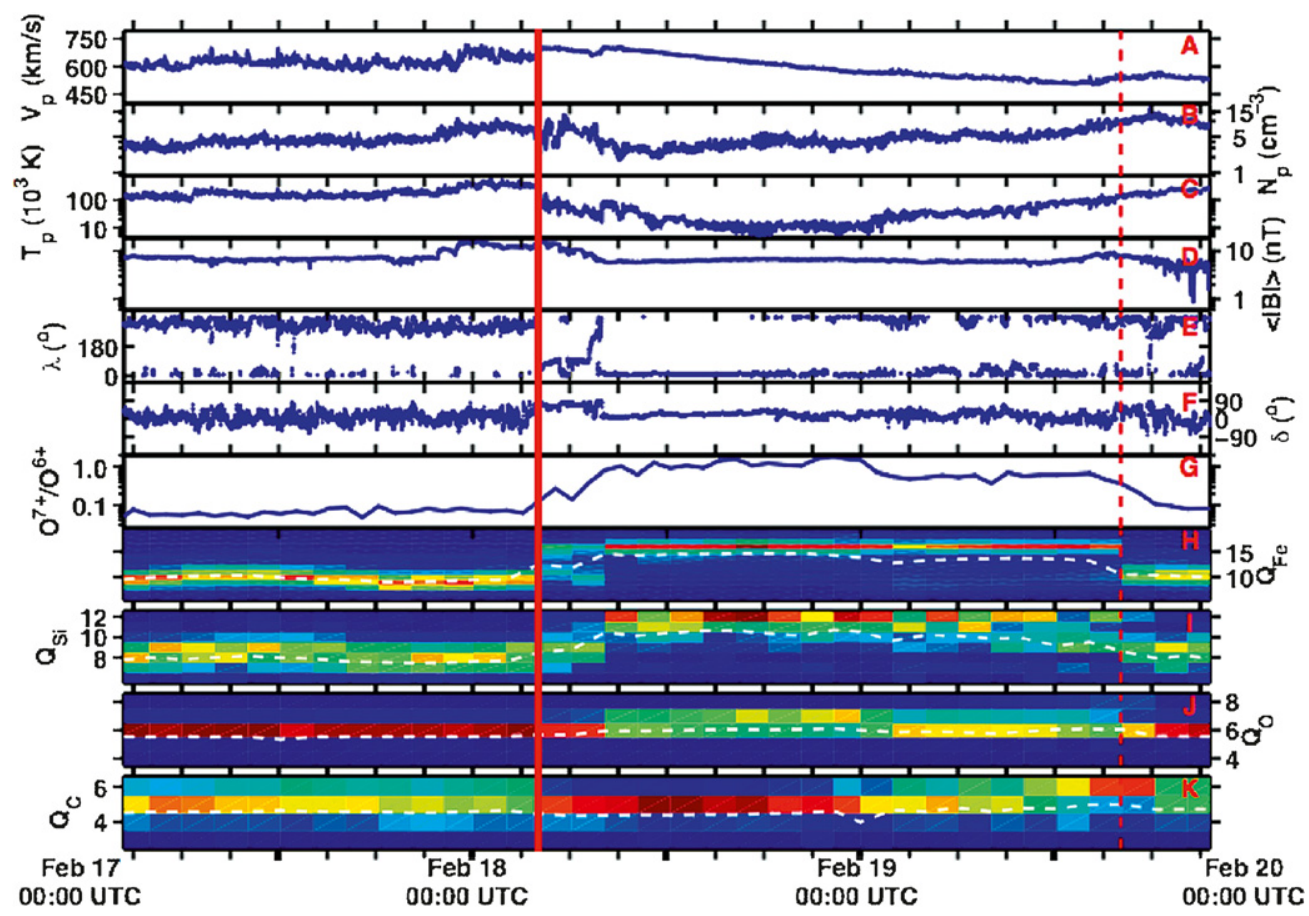

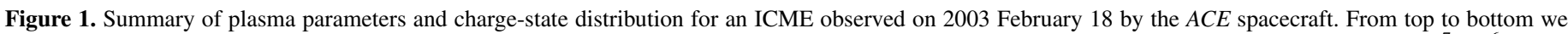

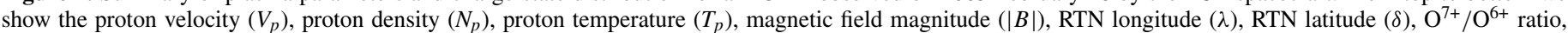

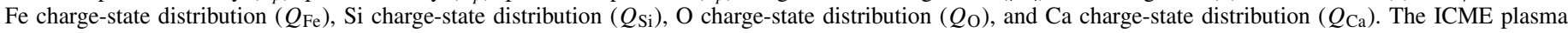

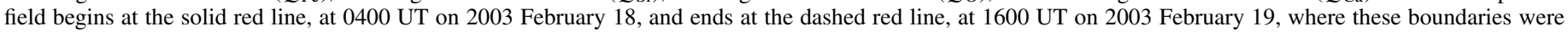
determined by Richardson \& Cane (2010). It can be seen that all four charge-state distributions experience an enhancement during the ICME interval.

(A color version of this figure is available in the online journal.)

The exact configuration of the observed ionization state is affected by collisional ionization, from electrons colliding with the ions in the plasma, and excitation autoionization, where electrons from the outermost shells are lost due to the relaxation of an inner shell electron (Cowan \& Mann 1979; Hundhausen et al. 1968; Hundhausen 1972). The observed ionization states are an important indicator of the CME's thermal environment close to the Sun. They are formed through a freeze-in process within 1-5 $R_{\odot}$ (Bame et al. 1974; Buergi \& Geiss 1986; Hundhausen et al. 1968; Hundhausen 1972). Very close to the Sun, while timescales for recombination and ionization are much shorter than the plasma's expansion timescale, the ionization states of heavy ions are approximately in ionization equilibrium, reflecting the local electron temperature. Due to the CME's expansion into the heliosphere, the electron density decreases rapidly and thus the recombination timescale increases as well. At a given heliocentric distance-depending on the specific characteristics of each ion and the electron environment - the ionic charge state freezes in and no longer changes. At this point, the charge state is said to be "frozen in." Due to the characteristic differences of atomic physics for each ion, the heliocentric distance where freeze-in occurs varies widely, and simultaneously observed charge-state distributions become a sensitive measure for the thermal evolution of the CME plasma near the Sun during the entire radial range where freeze-in occurs.

The unique nature of ICME charge states is further illustrated by Figure 2, showing a comparison of a time period of slow solar wind composition observed from 0000 UT to 0200 UT on 2003 July 30 with observation of an ICME from 0200 UT to 0400 UT on 2003 January 27. It is evident from Figure 2 that there is a noticeable increase in ionic charge states for each element in the ICME plasma compared to the typical wind. There is also a significant change in the qualitative nature of the distributions. Specifically (1) a significant fraction of $\mathrm{C}$ becomes fully ionized; (2) $\mathrm{O}$ shows large deviations from $\mathrm{O}^{6+}$, a He-like charge state that dominates solar wind measurements; (3) Si charge states are substantially increased for the ICME period and peak at $\mathrm{Si}^{12+}$, the He-like charge state; and (4) Fe transitions into a bimodal charge distribution peaked around $\mathrm{Fe}^{10+}$ and $\mathrm{Fe}^{16+}$, the Ne-like charge state discussed earlier. Such bi-modal charge distributions have been anecdotally reported before, but it is not generally known how common they are within ICMEs.

The purpose of this paper is twofold. First, we survey ionic charge states in ICME plasma to determine the rate of occurrence of bi-modal Fe charge-state distributions. Second, we develop a model to determine physical constraints for the ICME expansion especially focused on bi-modal Fe charge-state distributions, while simultaneously, qualitatively re-creating the $\mathrm{C}, \mathrm{O}$, and $\mathrm{Si}$ observations. Using this methodology, we will develop constraints for the temperature and density evolution of CMEs near the Sun.

\section{OBSERVATIONS}

This research is enabled, in part, by plasma observations from composition sensors such as the Solar Wind Ion Composition Spectrometer (SWICS) on board ACE (Gloeckler et al. 1998). These instruments independently determine the speed, mass and charge of incident ions and therefore enable the measurement of full charge distributions in ICMEs. The time resolutions of these measurements are limited by counting statistics of incident ions, and are typically limited to $2 \mathrm{hr}$ for $A C E-S W I C S$. The $\mathrm{O}^{7+} / \mathrm{O}^{6+}$ ratio is typically measured with 

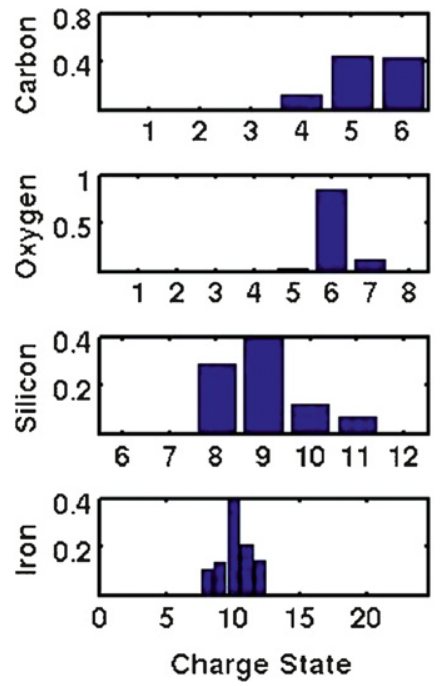

Typical Solar Wind Plasma
CME Plasma
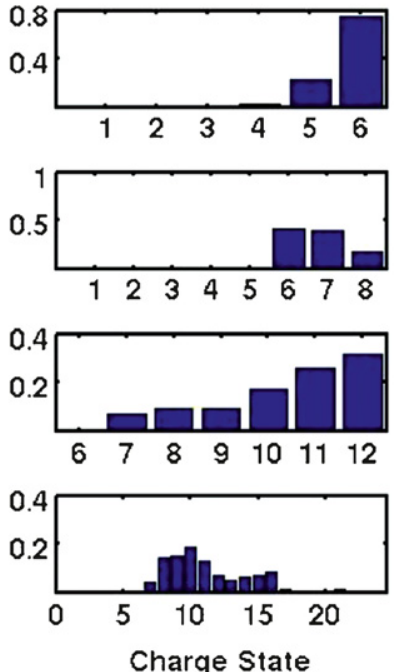

Figure 2. Comparison of the slow solar wind (left) and of ICME plasma (right). The ICME plasma shows enhanced charge states for all four atomic species compared to the typical solar wind. Note the bi-modal structure of Fe with peaks at $\mathrm{Fe}^{10+}$ and $\mathrm{Fe}^{16+}$. For more details, refer to the text.

(A color version of this figure is available in the online journal.)

$1 \mathrm{hr}$ time resolution. For each time period, ions are accumulated and analyzed largely using the methodology described by von Steiger et al. (2000). The analysis of Fe charge states is rather straightforward because its mass of approximately 56 amu substantially exceeds the mass of other heavy ions of similar abundance in the solar wind. $\mathrm{C}$ and $\mathrm{O}$ are the most abundant heavy ions in the wind and are therefore easily discernible. Our Si measurements currently are limited to charge states between $8+$ and $12+$ due to significant overlap with other neighboring ions in time of flight and energy space. Using these data, charge-state calculations are accurate approximately $99 \%$ of the time. Data gaps occur due to low counting statistics or operational events on $A C E$ independent of the solar wind plasma and compositional properties.

The analysis of each ICME includes composition data, as well as plasma and field observations, as demonstrated in Figure 1. The proton moments obtained by the Solar Wind Electron, Proton and Alpha Monitor (SWEPAM; McComas et al. 1998) are averaged over a $64 \mathrm{~s}$ time period. The magnetic field observations are provided by the Magnetic Field Experiment (MAG; Smith et al. 1998), averaged over $64 \mathrm{~s}$ intervals as well. ICME times and plasma boundaries are taken from Richardson \& Cane (2010), which were determined primarily by magnetic and plasma signatures, while using composition and chargestate data to confirm identification.

Richardson \& Cane (2004) have previously discussed the observations of generally enhanced ionization of $\mathrm{C}$ and $\mathrm{O}$ within ICMEs as compared to solar wind of the same speed. Indeed, such signatures were observed in 70\%-90\% of all CMEs irrespective of their field configuration. ICMEs associated with a magnetic cloud are shown to have a higher concurrence of compositional anomalies than non-cloud ICMEs.

Here, we focus on the bi-modal nature of Fe distribution, generally with peaks at $\mathrm{Fe}^{10+}$ and $\mathrm{Fe}^{16+}$. Inspecting the $A C E$ data, it is easily observed that bi-modal Fe charge states are often present in ICMEs. However, these signatures often do not extend throughout the event. For example, bi-modal distributions are observed in Figure 1 during the first four hours from 0400 UT to 0800 UT. The bi-modal nature of the ICME is not easily discernable from time 0800 UT until the beginning of February 19 , but is seen again from then on until the end of the ICME passage at 1600 UT. In contrast, bi-modal Fe charge states are equally likely to be observed during any time periods within ICMEs.

Figure 3 shows ACE observations of a second ICME, observed on 2003 January 27, presented in a format identical to that of Figure 1. The ICME boundaries were once again taken from the study of Richardson \& Cane (2010).

The plasma composition during this ICME looks qualitatively similar to the event shown in Figure 1: all four ionic distributions show significant enhancement during the event leading to higher than average charge states, indicative of temperature enhancements. Also, the Fe charge-state distribution is bi-modal for a majority of the ICME's duration.

It is well known that ICMEs often include identifiable subparts (Mulligan \& Russell 2001), with each having specific magnetic field configurations or plasma characteristics with well-defined transitions between them. The ICME in Figure 3 includes such a qualitative transition at time 1600 UT on January 27. Prior to this, the magnetic field configuration is that of a magnetic flux rope with the characteristic suppressed magnetic field turbulence, and reduced density characteristic of a magnetic cloud (Klein \& Burlaga 1982; Richardson et al. 2000; Zurbuchen \& Richardson 2006). The plasma temperature exhibits some interesting sub-structure during this time period. At 1600 UT, we observe a distinct transition visible in all plasma quantities-except for the plasma velocity. After 1700 UT, the magnetic field is again rather smooth; however, its axis has changed direction.

The compositional signatures appear to reflect this transition as well. SWICS does not have a high enough time resolution to analyze this change in compositional signatures with the same time accuracy as SWEPAM and MAG; however, there are discernible differences between the compositional signatures before and after $1600 \mathrm{UT}$. The heavy ion composition during the first part of the ICME has substructures which have qualitative correspondence with variability in plasma temperature. It has also been argued that this plasma heating is strongly associated with flares (Lepri \& Zurbuchen 2004; Rakowski et al. 2007; Reinard 2005). Based on this observation, as well as analogous observations in Figure 1, and in many other ICMEs studied, we conclude that compositional structure reflects the plasma structure and topology in ejecta.

An interesting question to ask is how prevalent are bi-modal Fe distributions in ICME plasmas. We address this by surveying all ICMEs from 2000 until 2007 that were identified and characterized by Richardson \& Cane (2010). For each ICME in this time period, the percentage of bi-modal plasma is determined. This percentage is defined as the ratio of the time with bi-modal Fe observations divided by the total time during the ICME period during which composition data exist. The bi-modal characteristic is assumed to be present if there was a significant minimum in the ionic charge distribution, which includes more than one single charge state. This limits spurious minima caused by poor statistics in the data.

Figure 4 shows the results of this survey, including approximately 200 ICMEs. The top panel of the figure shows the cumulative probability distribution function (CDF) computed from the bottom panel, which shows a probability distribution of ICMEs according to the fraction that is observed in the bimodal state normalized to one. It is immediately noticeable that 


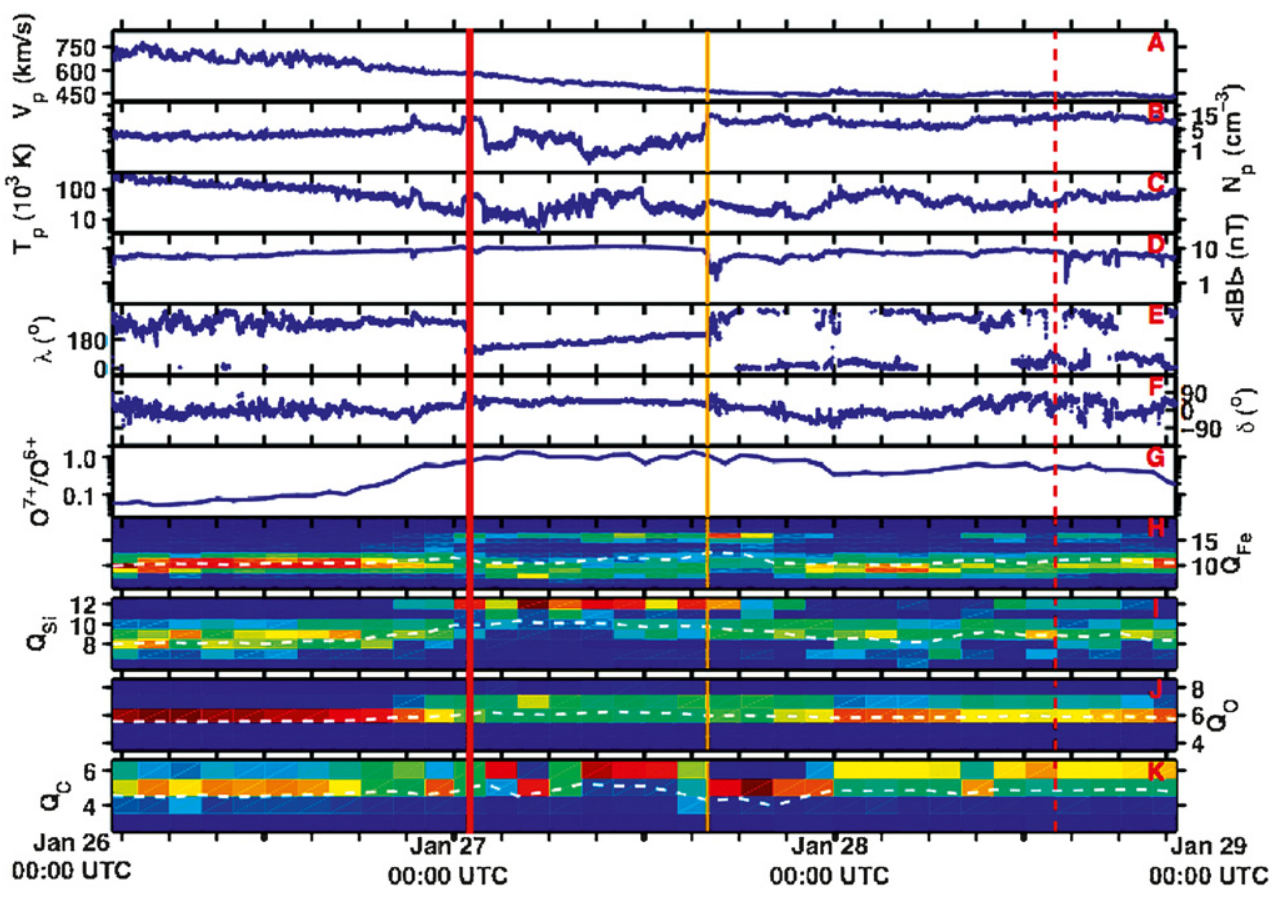

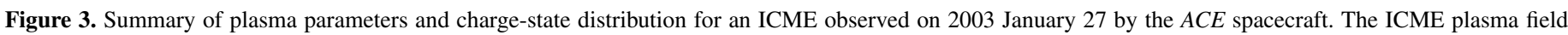

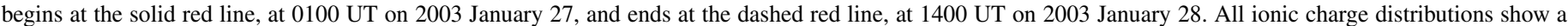

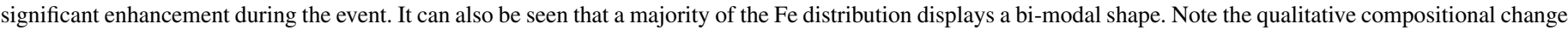

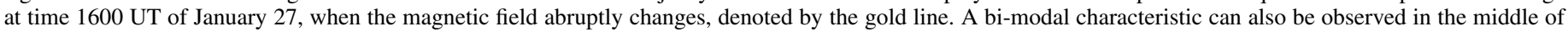
day 28.

(A color version of this figure is available in the online journal.)

more than $95 \%$ of ICMEs in this study include a measurable fraction of bi-modal Fe plasma. This finding also indicates that 95\% of ICMEs exhibit elevated Fe charge states within their boundaries. High charge states in ICMEs are therefore much more ubiquitous than reported in previous studies, which indicated that the prevalence of high charge states was 50\% (Lepri \& Zurbuchen 2004; Lepri et al. 2001). Using the CDF plot in the top panel, it can also be seen that $50 \%$ of ICMEs have at least $55 \%$ bi-modal Fe charge distributions. This bi-modal condition is thus found to be common in nearly all ICMEs and therefore becomes a critical observational criterion for our understanding of thermal properties of all CMEs near the Sun.

It has been previously argued that bi-modal Fe observations are the result of plasma mixing between hot and cold populations (Gibson et al. 2006; Gopalswamy et al. 2001; Rakowski et al. 2007). However, our analysis together with the qualitative analysis of transitions in ICMEs suggests that bi-modal charge distributions are an intrinsic property of a given plasma flow and not likely a mixing signature, which might be more diffusive in nature and more focused on specific boundaries and locales between cold and hot parts of a given CME. Using a simple ionization model, we show that bi-modal charge states can result from a rapidly heated plasma close to the Sun, which then cools as the plasma expands moving downstream. If the bi-modal distribution were truly a mixing signature, one would not expect to observe it nearly as often as we have shown in Figure 2. The nearly ubiquitous presence of some bi-modal material suggests that its distribution is a result of an inherent property of the plasma and not a mixing phenomenon.

\section{MODEL}

The authors have developed a freeze-in code, which solves for the final charge-state distribution of a given atomic species

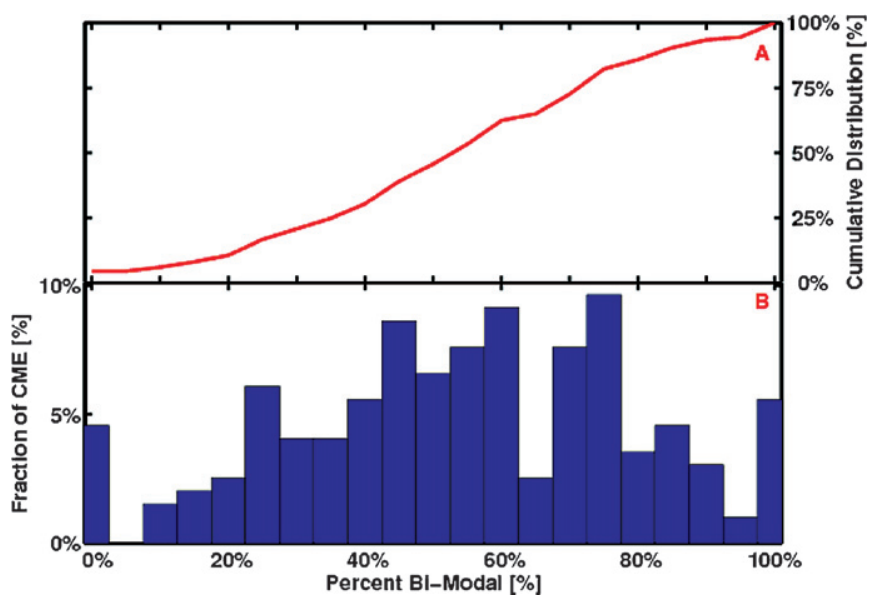

Figure 4. Statistical survey of bi-modal Fe distributions in ICME plasma. The bottom panel is a histogram showing the results of the survey. Percentage of total CMEs investigated is on the vertical axis, while percentage of bi-modal plasma in the CME is on the horizontal axis. The top panel shows a CDF of the resulting data set. This vertical axis is the probability that a given CME will have at least percentage of bi-modal Fe in the plasma field as the corresponding percentage on the horizontal axis.

(A color version of this figure is available in the online journal.)

under simple assumptions on a given CME's heating and expansion properties. To obtain the relative density of a given ion, $y_{i}=n_{i} / \sum_{i=0}^{Z} n_{i}$, and in particular, the freeze-in condition, the following continuity equation, valid in the rest frame of the ejected CME (Ko et al. 1997), is solved:

$\frac{\partial n_{i}}{\partial t}=n_{e}\left(y_{i-1} C_{i-1}\left(T_{e}\right)-y_{i}\left(C_{i}\left(T_{e}\right)+R_{i-1}\left(T_{e}\right)\right)+y_{i+1} R_{i}\left(T_{e}\right)\right)$. 
It is important to note that $n_{i}$ is the number density of the charge state $i$ of the atomic species of interest, while $y_{i}$ is the relative number density. To solve this equation, the ion-specific recombination rates, $R_{i}$, and ionization rates, $C_{i}$, from Mazzotta et al. (1998) are used. These rates include electron ionization and autoionization as well as radiative and dielectronic recombination under the assumption of locally Maxwellian electron distribution functions, which has been used as reasonable approximation in previous studies (e.g., Laming \& Lepri 2007). These depend only on the electron temperature $T_{e}$ and are valid for all temperatures greater than $10^{4} \mathrm{~K}$. Photoionization is neglected here, as it plays a significant role for only singly and some doubly charged ions (Marsch et al. 1995).

Equation (1) can be solved using a given electron density and temperature history $\left(n_{e}(t), T_{e}(t)\right)$, which can be assumed, or constrained by observations. The system of ordinary differential equations described by Equation (1) is generally stiff as recombination and ionization rates can vary over many orders of magnitude. To address this, we use a fourth-order Runge-Kutta method that is specially suited for stiff equations (Press et al. 2002). For computational efficiency, the method also uses an adaptive step size. At each time, the method solves for the number density of an atomic species ionized to the charge state $i$. We also check the accuracy of the integrator by testing the condition $\sum y_{i}=1$. Our integrations are accurate to better than $10^{-6}$.

To model the heating and expansion of the plasma using the equations above, we calculate the CME bulk parameters at each time $t$. We examine two types of evolution, one in which the CME plasma close to the corona is assumed to be in an elevated temperature and density state and then undergoes a pure adiabatic expansion. We show below that this evolution is not compatible with the composition data. The other evolution is one in which we include an initial rapid heating phase while the plasma is in the lower corona, and then impose the adiabatic expansion. Such a heating would be expected from the energy release due to the flare reconnection that forms the ejected CME flux rope in the breakout model (e.g., Lynch et al. 2004).

Since the heating of the ejected plasma is not well known from first-principle models, we assume a simple, ad hoc form for this heating that has the minimum possible parameters. The plasma is taken to have some initial plasma temperature $T_{0}$ at $t=0$, which rapidly increases to a specified maximum temperature $\left(T_{\max }\right)$, in a specified heliospheric distance $\left(r_{\text {heat }}\right)$. Generally, the heating distance is chosen to be some value between 0 and $0.5 R_{\odot}$ from the surface. In addition to the heating profile, some assumption must be made as to the evolution of the density. The simplest is to allow the density to evolve adiabatically; in other words, the heating is due to a pure compression of the plasma. Such a compression is, indeed, seen in simulations of $\mathrm{CME}$ /flare evolution. The reconnection jets coming out of the flare current sheet drive plasma compression both in the flare loop system remaining in the corona and in the escaping CME plasmoid. However, we also expect there to be some direct heating of the plasma due to magnetic dissipation throughout the CME/ICME evolution. This direct heating is highly modelspecific; therefore, to keep our results in this paper as general as possible, we consider only an adiabatic compression for the initial heating evolution, as well as an adiabatic expansion for the ejection. Of course, given some quantitative model for CME temperature and density evolution, it would be straightforward to use it, instead of the adiabatic assumption, in our analysis.
Equation (2) shows the forms of the temperature and density evolution that we use for the initial heating. Note that the model has only four free parameters: the initial temperature and density, the maximum temperature, and the radius at which this maximum temperature occurs. This is the minimum possible number of free parameters for any such model:

$$
\begin{aligned}
& \left.\begin{array}{l}
T(t)=\left(T_{\max }-T_{0}\right) \sin \left(2 \pi \frac{\left(\left[r(t) / R_{\mathrm{Sun}}\right]-1\right)}{4 *\left[r_{\text {heat }} / R_{\mathrm{Sun}}\right]}\right)+T_{0} \\
n(t)=n_{\max }\left(\frac{T(t)}{T_{\max }}\right)^{3 / 2}
\end{array}\right\}, \\
& \text { where } r(t) \leqslant r_{\text {heat }} \text {. }
\end{aligned}
$$

The particular functional form for the temperature evolution, a quarter sinusoid, was used to qualitatively match the rapid heating near the corona, but its exact shape is not critical to create bi-modal distributions. Additionally, it ensures that the temperature has a continuous derivative, which is necessary for greater accuracy of the numerical method. The evolution of the density is derived directly from the adiabatic expansion formula, assuming a monoatomic ideal gas. After the rapid heating phase, the plasma cools as its volume expands. Again, the adiabatic equations couple the density and temperature evolution. Equation (3) describes the evolution of these parameters:

$$
\left.\begin{array}{l}
T(t)=T_{\max }\left(\frac{n(t)}{n_{\max }}\right)^{\gamma-1.0} \\
n(t)=n_{\max }\left(\frac{r_{\text {heat }}}{r(t)}\right)^{\beta}
\end{array}\right\}, \quad \text { where } r(t)>r_{\text {heat }} .
$$

Parameter $\beta$ is an expansion factor of the plasma, typically set to a value between two and three. The cooling is dependent on the adiabatic index, $\gamma$, which is set to $5 / 3$, the value for an ideal monoatomic gas. Note that $n_{\max }$ and $T_{\max }$ occur at the end of the heating period and define the beginning of the expansion cooling phase.

Motivated by observational characteristics of CMEs, our model allows for a non-constant velocity profile of the plasma. It is particularly important to model rapid acceleration of CMEs near the Sun. CMEs rapidly accelerate to a maximum velocity, which remains nearly constant, much like the velocity profile of the solar wind (Gallagher et al. 2003). Using LASCO coronagraph images, a linear acceleration can be determined for some CMEs (St. Cyr et al. 2000). The Solar and Heliospheric Observatory/LASCO (SOHO/LASCO) CME catalog makes these values available for CMEs when the calculation is possible. In our model, therefore, the CME plasma undergoes linear acceleration from the corona until the velocity reaches the observed in situ velocity, from $A C E$. After this, the velocity is set to the observed value and remains constant.

Note that the total mass of CME plasma is assumed to be constant during the evolution: there is no net gain or loss of particles. Furthermore, as the plasma is continually ionized, the free electrons remain in our plasma volume. This allows the plasma to experience as much recombination as is possible by the available electrons, creating quasi-local ionization and recombination. In addition, we have no specific requirements on the velocity distribution of the electrons. The model also assumes that all electrons have a Maxwellian distribution. Additionally, we initialize the charge state of our plasma from the local thermodynamic equilibrium solution from the ionization equations discussed earlier. Finally, the computed temperature is used to determine the ionization and recombination rates at each time step. 

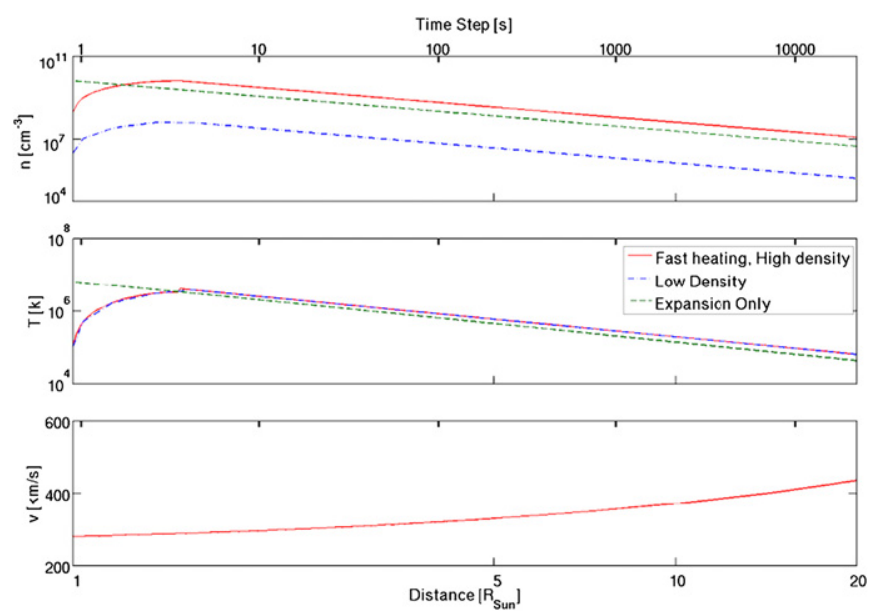

Figure 5. Bulk plasma parameters for the model run of the 2003 January 27 CME. From top to bottom volume, density, temperature, and velocity are shown The red line is the case with both fast heating and high density. The green dashed line is the case with only rapid heating, but a depressed density. Finally, the blue dash-dotted line is the case for no heating of the plasma, only the rapid expansion of the plasma.

(A color version of this figure is available in the online journal.)

To study the various physical characteristics of freeze-in within CMEs, we study a CME with an associated linear acceleration. This allows us to determine a velocity profile. Specifically, we use a velocity profile matching the 2003 January 27 CME determined from the linear acceleration (St. Cyr et al. 2000) and $A C E$ observations and whose velocity profile is shown in Figure 5. We then perform a parametric study to understand the temperature profiles that may lead to the observed characteristics. To demonstrate the impacts of the various model parameters on the freeze-in ionization rates, we perform a series of combinations of the following three characteristics, which we found to be crucial for this calculation: rapid heating, rapid expansion, and an elevated initial bulk density. We want to show that the absence of one of these key characteristics leads to qualitative disagreements to the observations summarized in Figure 2. The key assumptions of the three illustrative calculations are shown in Figure 5.

First, results are shown that include a with rapid heating and rapid expansion, but with relatively low initial density, close to typical solar wind densities (Wilhelm et al. 1998). Second, results from the case having rapid expansion and an enhanced bulk density are shown. However, no heating is present and the plasma only cools. Third, results are shown that include all three ingredients. This is the only case that reproduces, qualitatively, the observed freeze-in distributions. To determine the relative accuracy of these cases, we use the 2003 January 27 event shown in Figures 3; however, it should be noted that our model is not sophisticated enough to match the data quantitatively. We can only match the general characteristics of the observed ionic charges states of heavy ions, such as the Fe bi-modal distribution.

Figure 5 summarizes the basic assumptions for each set of input profiles used; different colored lines represent a different case. The top panel shows the density evolution for each model. The second panel shows the temperature evolution. Here, the red and blue dash-dotted lines are on top of each other, since they both experience rapid heating, while the green dashed line diverges initially. The initial temperature of the cooling case was chosen such that it becomes similar to the temperature curves of the other cases downstream of the corona. This was done to

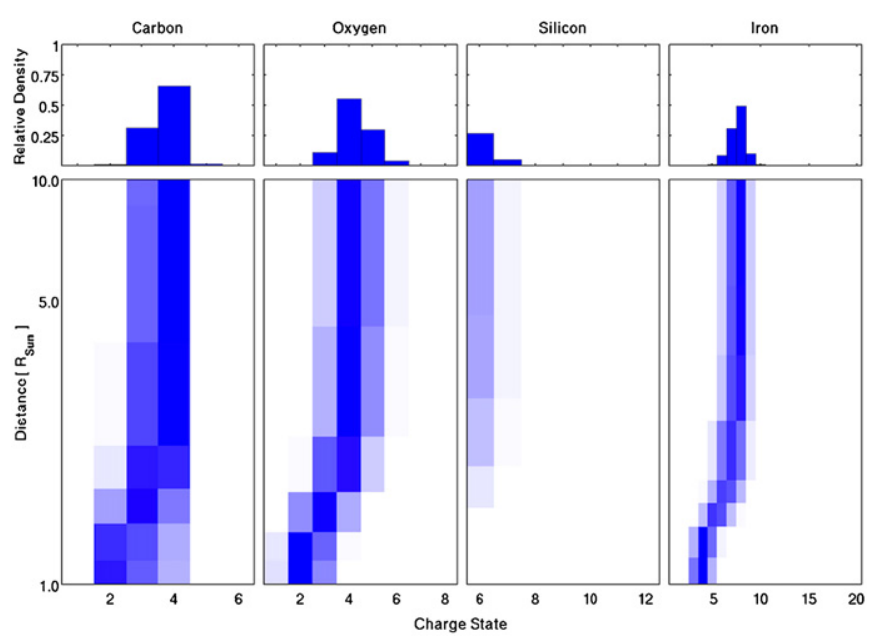

Figure 6. Results from the model which includes rapid heating and expansion, but with the omission of an enhanced initial density. The bottom set of panels shows the evolution of the charge states from the coronal surface to a distance of $10 R_{\odot}$ away plotted for arbitrary instances during the expansion. For all four atomic species, it can be seen that freeze-in occurred around $3 R_{\odot}$. The top panels show the frozen-in charge-state distribution observed at $1 \mathrm{AU}$.

(A color version of this figure is available in the online journal.)

ensure that any difference in results was due only to the near coronal heating profile and not to any other portions of the temperature profile. It is also important to note that the shape of the temperature curve of each case qualitatively matches the shape of its respective density curve. This is a result of the temperature and density evolutions being tied together by the adiabatic relation. Finally, the last panel shows the velocity, which is identical for all three cases, as this curve was determined from LASCO and $A C E$ observations of this particular CME by the method described above. Below, we show the final charge-state results and evolution for these three model cases.

\section{RESULTS AND DISCUSSION}

As discussed in the previous section, we ran our freeze-in and CME expansion model for three different cases attempting to recreate the charge-state observations from the 2003 January 27 CME (Figure 3). The first case simulates a CME plasma that undergoes rapid heating near the corona, along with rapid expansion of its volume. However, the initial electron bulk density is low, $6 \times 10^{7} \mathrm{~cm}^{-3}$, which is in qualitative agreement with nominal solar wind densities at the corona (Wilhelm et al. 1998). Figure 6 shows the resulting charge-state distributions of four atomic species $(\mathrm{C}, \mathrm{O}, \mathrm{Si}$, and $\mathrm{Fe}$ ) plotted at specific time intervals during the expansion. Expansion times were translated into radial scale using the integrated velocity profile shown in Figure 5.

The bottom four panels of Figure 6 show the evolution of the charge-state distribution, for each species, from the coronal surface to a distance of $10 R_{\odot}$, after which the ionic charge states remain frozen-in for all considered cases. In fact, charge states are generally frozen-in within a distance of $5 R_{\odot}$ (Buergi \& Geiss 1986; Hundhausen 1972), and our model agrees with this result showing the charge states to be frozen-in at a distance of approximately $3 R_{\odot}$. After this point, the charge-state distribution remains constant. The top series of panels show the final resulting and frozen-in charge-state distributions observed at $1 \mathrm{AU}$, the approximate location of in situ composition observations. It is immediately apparent, 


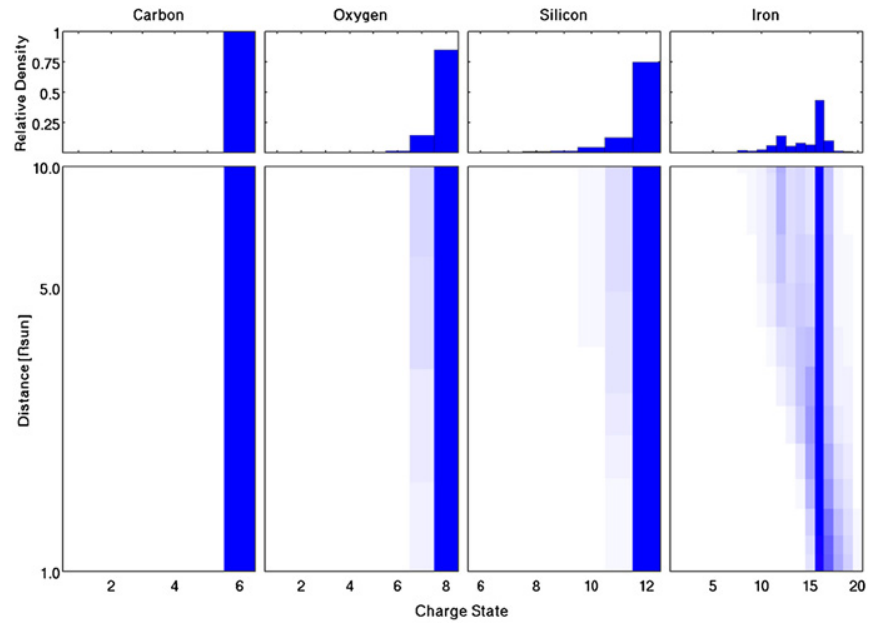

Figure 7. Results from the model which includes rapid expansion and also has a sufficiently large initial bulk density, but omits rapid heating. Format as in Figure 6.

(A color version of this figure is available in the online journal.)

based on the data in Figure 2 and discussed in Section 2, that the resulting charge states are much too cool. Without a significant density increase over solar wind profiles, the resulting ionic charge distributions are not sufficiently ionized, leaving the charge states of all the species at values much lower than are observed in ICMEs.

In Figure 7, we present the resulting charge-state distribution from the case with initial high density and initial high temperature which only models the rapid expansion of an ICME.

It is evident that the resulting plasma in this case of pure expansion has more enhanced charge states for all atomic species than the previous low-density case. The problem, however, is that the charge states at $1 \mathrm{AU}$ are much hotter than is observed. The $\mathrm{C}, \mathrm{O}$, and $\mathrm{Si}$ are all essentially fully ionized. The reason is that the plasma has simply not had enough time to recombine down to the observed charge states due to the rapid expansion and accompanying density decrease. We note that the Fe distribution does exhibit bi-modality, but the peak at $\mathrm{Fe}^{16+}$ is much stronger than that at $\mathrm{Fe}^{10+}$, which does not agree with observations. These results suggest that rapid expansion combined with high initial density may be critical ingredients for producing bi-modal Fe distributions, but it is unlikely that these two ingredients by themselves cannot be made consistent with all the compositional data.

Finally, in Figure 8 we present the case where all three ingredients (high density, rapid coronal heating, and rapid expansion) are present in the model.

This case also shows a freeze-in behavior comparable to the previous case. Again, charge states freeze in within the first few solar radii from the corona, and the model matches the CME-like states of $\mathrm{C}$ and $\mathrm{O}$ as well as key characteristics of Si. However, under this set of assumptions, a qualitatively similar bi-modal Fe distribution results, creating a consistent set of results under the assumption of a single density, temperature, and velocity profile. Specifically, enhanced $\mathrm{C}$ and $\mathrm{O}$ charge states are seen, yet neither are fully ionized. Additionally, we see enhanced silicon charge states, and two distinct peaks in iron at $\mathrm{Fe}^{10+}$ and $\mathrm{Fe}^{16+}$. The formation of bi-modal iron peaks is in part a result of high initial electron density and rapid expansion along with rapid heating, as can be seen by comparing Figures 7 and 8 .

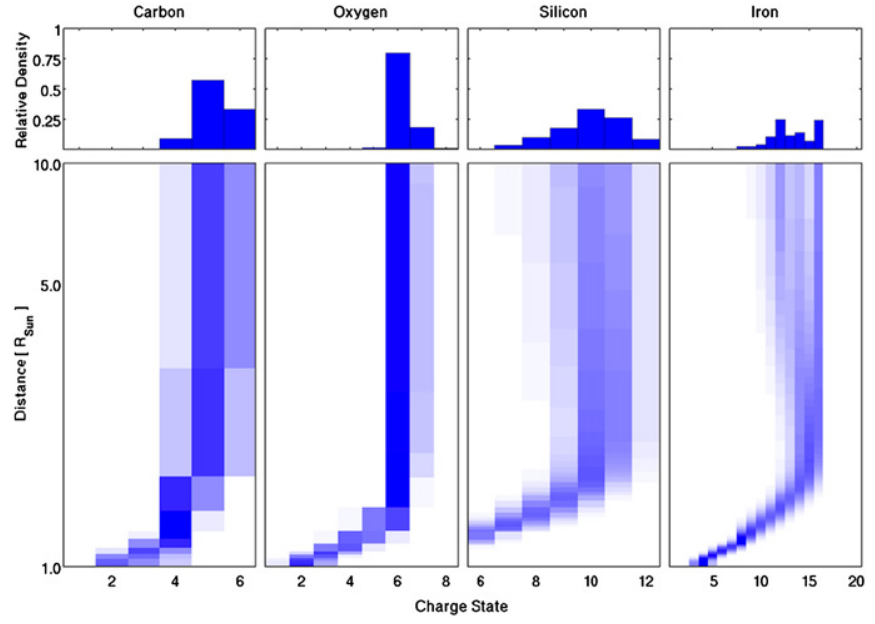

Figure 8. Results from the model for the case where the plasma is rapidly heated, experiences rapid expansion, and has a sufficiently high initial density. Format as in Figure 6.

(A color version of this figure is available in the online journal.)

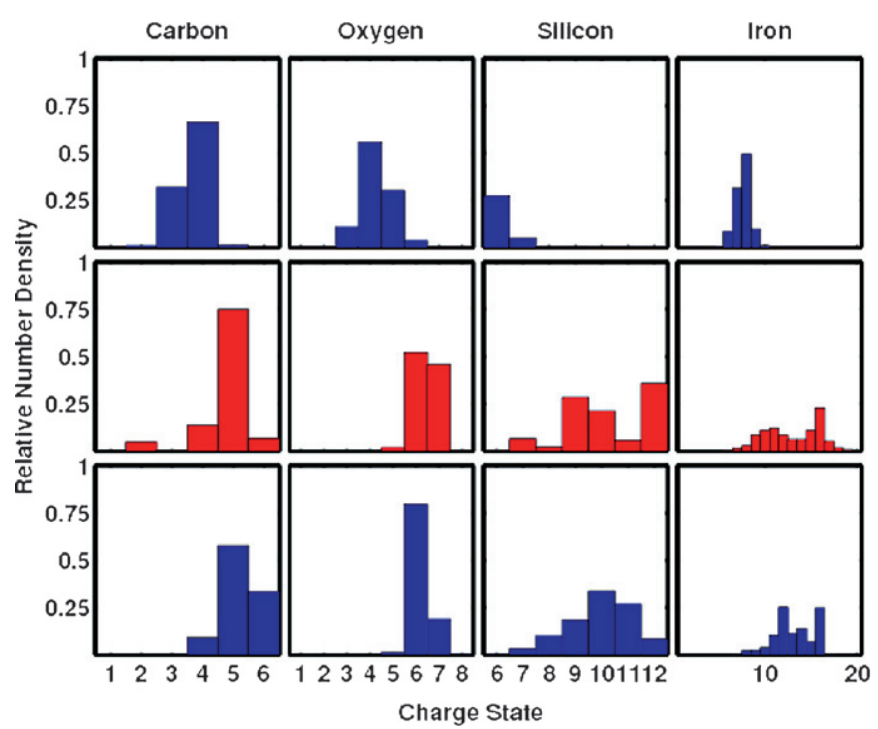

Figure 9. Charge state distributions for carbon, oxygen, silicon, and iron. The top row is the final distribution result from the model in Figure 6 . The middle row is the observed $A C E$ /SWICS charge-state distribution for a $2 \mathrm{hr}$ span on the 2003 January 27 . The final row is the final distribution from the model in Figure 8 .

(A color version of this figure is available in the online journal.)

Figure 9 compares the results in Figures 6 and 8 with representative observations of the 2003 January $27 \mathrm{CME}$ from 0100 to 1400 hour on January 28, which is representative of the bi-modal distribution usually observed.

The top row of charge-state distribution in Figure 9 shows the result of the model that had rapid heating and expansion, but lacked a high initial density, the results of the case shown in Figure 6. The bottom row, from Figure 8, shows the result when the density is high, and rapid heating and expansion are present. We left out the case shown in Figure 7 because peaks are generally in the same place, and only the relative number density in the peaks differs. In addition, the Fe distribution of Figure 7 is primarily unimodal. The middle row shows the actual chargestate distribution observations from ACE/SWICS of the 2003 January 27 ICME, which we are modeling here. All the charge states in the top line are much lower than what is observed, 
while the relative shapes of the results in the bottom row are in qualitative agreement with observations, perhaps to a lesser degree for Si. Silicon from our model has a single peak, around charge states $\mathrm{Si}^{9+, 10+}$, while $A C E$ observes a bi-modal silicon distribution with peaks at $\mathrm{Si}^{9+}$ and $\mathrm{Si}^{12+}$. Our model results in a considerable amount of $\mathrm{Si}^{11+}$, which would be ionized further if the temperature curve was modified slightly to have a shorter heating period. Finally, experimental issues limiting Si data were already discussed previously and may also effect this comparison.

In order to test the robustness of our model's code, we verified that a population of hydrogen would be fully ionized, using the assumptions made for the third case. It was found that hydrogen became fully ionized, as expected. Additionally, we repeated this test for helium. Again, we found that helium becomes fully ionized; however, a small population recombines in the $\mathrm{He}^{+}$population, $10^{-4}$ in relative density. This is due to the model's assumptions, as this small recombination only occurs once the temperature of the plasma drops below the range of valid temperatures for the reaction rates. As previously stated, the rates used in the model are valid only for temperatures greater than $10^{4} \mathrm{~K}$ (Mazzotta et al. 1998); however, during the full evolution of our plasma, the temperature does fall below this value.

We also conducted tests to put a limit on how much the maximum density can vary with the particular temperature profile chosen. For the third case, which successfully recreated a bi-modal $\mathrm{Fe}$ distribution while matching $\mathrm{C}$ and $\mathrm{O}$ distributions, an initial electron density of $6.0 \times 10^{9}\left(\mathrm{~cm}^{-3}\right)$ was used. By incrementing this value up and down we were able to determine a range of valid densities resulting in the Fe bi-modal distribution, which is $4.75 \times 10^{9} \mathrm{~cm}^{-3} \leqslant n_{e} \leqslant 8.5 \times 10^{9} \mathrm{~cm}^{-3}$.

\section{CONCLUSIONS}

The presence of high charge-state ions in the solar wind is known to be a sufficient condition for identification of ICMEs, and was previously thought to exist in $<70 \%$ of ICMEs. The current study reveals that $95 \%$ of ICMEs in fact have high ionic charge states, exhibiting bi-modal characteristics in Fe. These findings reinforce the crucial role heavy ion composition signatures play in identifying ICME material. The pervasive nature of the observed ICME charge-state characteristics at $1 \mathrm{AU}$ (Lepri et al. 2001; Richardson \& Cane 2010) suggests a simple and ubiquitous physical process or explanation. From running a large number of test models, we conclude that charge-state distributions are powerful tools for constraining the thermal properties of CME plasma near the Sun. We find that bi-modal Fe charge distributions can evolve from a plasma that rapidly expands from a high initial density. We also find that bi-modal Fe observations and elevated Si observations can only be made consistent with concurrent $\mathrm{C}$ and $\mathrm{O}$ data, if the elevated initial density goes through a rapid heating before it cools in the expansion. No further assumptions are needed to fit qualitatively these data.

Based on our simulations, we also believe that there is a robustness to this result. For example, Neukomm \& Bochsler (1996) focus on the evolution of charge-state distributions of heavy ions in closed magnetic structures. Although they did not explore their models at sufficiently high temperatures, we see that certain key processes are consistent.

For example, the model suggests the importance of noble gas-like charge states, such as $\mathrm{O}^{6+}$ and $\mathrm{Fe}^{16+}$. These ionic states have recombination rates that are substantially smaller than their adjacent charge states. Thus, these charge states freeze in earlier than their adjacent charge states, such as $\mathrm{Fe}^{15+}$. Thus, subsequent cooling of initially hot plasma will tend to have $\mathrm{Fe}^{16+}$ to remain prevalent, whereas $\mathrm{Fe}^{15+}$ can further cascade to lower states, such as $14+, 13+$, etc. This is the inherent cause for the dual-peaked nature of the observed $\mathrm{Fe}$ charge characteristic, and thus expected to be a robust result, independent of the details of the heating and cooling of the plasma.

We cannot exclude the possibility that other candidate processes, such as non-thermal electron characteristics near the Sun, or more complex evolutions involving multiple sources of plasma can achieve similar agreement with observations. We would argue, however, that the basic plasma evolution derived in this work-a rapid compression followed by an expansion-is the simplest and most likely explanation for the in situ observations. In order to observe $\mathrm{Fe}^{16+}$ at $1 \mathrm{AU}$, the plasma must reach fairly high temperatures, several $\mathrm{MK}$, before the radius at which the freeze-in condition sets in. But if the plasma is in thermal equilibrium at this point, then the resulting charge states for the lighter elements would be too high at $1 \mathrm{AU}$, as in Figure 7. The only way to avoid this discrepancy is to heat the plasma rapidly so that the lighter elements never reach their fully ionized state, while the iron achieves high ionization. Furthermore, since the velocity and expansion properties of CMEs are fairly accurately known from coronagraph observations, the observed charge-state distributions impose tight constraints on the plasma density during this heating and expansion evolution. Too low a density implies that the various elements never reach the observed ionization states, whereas too high washes out the bi-modal peak in iron. These straightforward and compelling arguments demonstrate the power of our compositional analysis techniques for deriving the detailed properties of the solar origins of space weather from in situ measurements in the heliosphere.

Note the evolution determined by our composition analysis: a rapid compressive heating to high density followed by expansion cooling is fully consistent with the expected effects of flare energy release on the thermodynamics of CME plasma (Harrison 1995; Vršnak et al. 2005). These effects have been discussed by many authors (e.g., Canfield \& Reardon 1998; Li et al. 2008) and typically relate to reconnection processes going on near the Sun (Lynch et al. 2004). The upward directed reconnection jets from the flare current sheet are expected to compress and heat the CME plasma, as derived above. By combining our data and modeling techniques with sophisticated MHD simulations (Antiochos et al. 1999; Gombosi et al. 2000; Linker et al. 1990), we can obtain a powerful tool for the analysis of CMEs and their evolution near the Sun. The computations presented in Figure 8 provide predictions of the presence of certain ions at specific times during the CME's evolution and should be testable, especially by forward modeling in which the calculation of the charge-state evolution is included in the MHD simulation. A key point is that the various models for CME onset predict different locations for the initial heating of the coronal plasma that is ejected into the heliosphere as an ICME (e.g., Forbes et al. 2006). Of course, many of these differences are expected to be washed out during the propagation to $1 \mathrm{AU}$; however, with the advent of composition measurements near the Sun from missions such as the Solar Orbiter, our analysis may be able to determine not only the coronal evolution of CME plasma, but the fundamental mechanism responsible for the eruption itself. 
This work used the SOHO/LASCO CME catalog developed by NASA, NRL, and the Catholic University of America. $\mathrm{SOHO}$ is a project of international cooperation between ESA and NASA. This work was performed, in part, with support from NASA grants NNX08AI11G, NNX07AB99G, and NNX08AM64G, as well as the GSRP program. T.H.Z. acknowledges the hospitality of the International Space Science Institute, where much of this work was performed. S.K.A. was supported, in part by the NASA HTP and TR\&T Programs. The authors thank Eric Christian for helpful discussions and comments on the manuscript. The authors thank Katherine Baldwin and Jim Raines for their early work, which was a foundation for this study.

\section{REFERENCES}

Antiochos, S. K., DeVore, C. R., \& Klimchuk, J. A. 1999, ApJ, 510, 485

Bame, S. J., Asbridge, J. R., Feldman, W. C., Fenimore, E. E., \& Gosling, J. T. 1979, Sol. Phys., 62, 179

Bame, S. J., Asbridge, J. R., Feldman, W. C., \& Kearney, P. D. 1974, Sol. Phys., 35,137

Brueckner, G. E., et al. 1995, Sol. Phys., 162, 357

Buergi, A., \& Geiss, J. 1986, Sol. Phys., 103, 347

Burlaga, L., Sittler, E., Mariani, F., \& Schwenn, R. 1981, J. Geophys. Res., 86, 6673

Canfield, R. C., \& Reardon, K. P. 1998, Sol. Phys., 182, 145

Cowan, R. D., \& Mann, J. B. 1979, ApJ, 232, 940

Dauphin, C., Vilmer, N., \& Krucker, S. 2006, A\&A, 455, 339

Forbes, T. G., et al. 2006, Space Sci. Rev., 123, 251

Gallagher, P. T., Lawrence, G. R., \& Dennis, B. R. 2003, ApJ, 588, L53

Gibson, S. E., Fan, Y., Török, T., \& Kliem, B. 2006, Space Sci. Rev., 124, 131

Gloeckler, G., et al. 1998, Space Sci. Rev., 86, 497

Goff, C. P., van Driel-Gesztelyi, L., Harra, L. K., Matthews, S. A., \& Mandrini, C. H. 2005, A\&A, 434, 761

Gombosi, T. I., DeZeeuw, D. L., Groth, C. P. T., Powell, K. G., \& Stout, Q. F. 2000, J. Atmos. Sol.-Terr. Phys., 62, 1515

Gopalswamy, N., Nunes, S., Yashiro, S., \& Howard, R. A. 2004, Adv. Space Res., 34, 391

Gopalswamy, N., Yashiro, S., Kaiser, M. L., Howard, R. A., \& Bougeret, J. L. 2001, ApJ, 548, L91

Gosling, J. T., Pizzo, V., \& Bame, S. J. 1973, J. Geophys. Res., 78, 2001

Harrison, R. A. 1995, A\&A, 304, 585

Henke, T., et al. 1998, Geophys. Res. Lett., 25, 3465

Hundhausen, A. J. 1972, Coronal Expansion and Solar Wind, Vol. 5 (Springer: Berlin)
Hundhausen, A. J., Gilbert, H. E., \& Bame, S. J. 1968, J. Geophys. Res., 73 5485

Klein, L. W., \& Burlaga, L. F. 1982, J. Geophys. Res., 87, 613

Ko, Y., Fisk, L. A., Geiss, J., Gloeckler, G., \& Guhathakurta, M. 1997, Sol. Phys., 171,345

Krucker, S., Hudson, H. S., Glesener, L., White, S. M., Masuda, S., Wuelser, J. P., \& Lin, R. P. 2010, ApJ, 714, 1108

Laming, J. M., \& Lepri, S. T. 2006, ApJ, 660, 1642

Lepri, S. T., \& Zurbuchen, T. H. 2004, J. Geophys. Res., 109, A01112

Lepri, S. T., Zurbuchen, T. H., Fisk, L. A., Richardson, I. G., Cane, H. V., \& Gloeckler, G. 2001, J. Geophys. Res., 106, 29231

Li, Y., Lynch, B. J., Stenborg, G., Luhmann, J. G., Huttunen, K. E. J., Welsch, B. T., Liewer, P. C., \& Vourlidas, A. 2008, ApJ, 681, L37

Lin, R. P., et al. 2003, ApJ, 595, L69

Linker, J. A., van Hoven, G., \& Schnack, D. D. 1990, J. Geophys. Res., 95, 4229

Lynch, B. J., Antiochos, S. K., MacNeice, P. J., Zurbuchen, T. H., \& Fisk, L. A. 2004, ApJ, 617, 589

Marsch, E., von Steiger, R., \& Bochsler, P. 1995, A\&A, 301, 261

Mazzotta, P., Mazzitelli, G., Colafransesco, S., \& Vittorio, N. 1998, A\&AS, 133,403

McComas, D. J., Bame, S. J., Barker, P., Feldman, W. C., Phillips, J. L., Riley, P., \& Griffee, J. W. 1998, Space Sci. Rev., 86, 563

Mulligan, T., \& Russell, C. T. 2001, J. Geophys. Res., 106, 10581

Neukomm, R. O., \& Bochsler, P. 1996, ApJ, 465, 462

Press, W. H., Teukolsky, S. A., Vetterling, W. T., \& Flannery, B. P. 2002, Numerical Recipes in C++ (2nd ed.; Cambridge: Cambridge Univ. Press)

Rakowski, C. E., Martin Laming, J., \& Lepri, S. T. 2007, ApJ, 667, 602

Reinard, A. 2005, ApJ, 620, 501

Richardson, I. G., Berdichevsky, D., Desch, M. D., \& Farrugia, C. J. 2000, Geophys. Res. Lett., 27, 3761

Richardson, I. G., \& Cane, H. V. 1995, J. Geophys. Res., 100, 23397

Richardson, I. G., \& Cane, H. V. 2004, J. Geophys. Res., 109, A09104

Richardson, I. G., \& Cane, H. V. 2010, Sol. Phys., 264, 189

Rodriguez, L., Woch, J., Krupp, N., Franz, M., von Steiger, R., Forsyth, R. J., Reisenfeld, D. B., \& Glasmeier, K. H. 2004, J. Geophys. Res., 109, A01108

Smith, C. W., L'Heureux, J., Ness, N. F., Acuña, M. H., Burlaga, L. F., \& Scheifele, J. 1998, Space Sci. Rev., 86, 613

St. Cyr, O. C., et al. 2000, J. Geophys. Res., 105, 18169

Stone, E. C., Frandsen, A. M., Mewaldt, R. A., Christian, E. R., Margolies, D., Ormes, J. F., \& Snow, F. 1998, Space Sci. Rev., 86, 1

Temmer, M., Veronig, A. M., Kontar, E. P., Krucker, S., \& Vršnak, B. 2010, ApJ, 712,1410

von Steiger, R., et al. 2000, J. Geophys. Res., 105, 27217

Vršnak, B., Sudar, D., \& Ruždjak, D. 2005, A\&A, 435, 1149

Wilhelm, K., Marsch, E., Dwivedi, B. N., Hassler, D. M., Lemaire, P., Gabriel, A. H., \& Martin, M. C. E. 1998, ApJ, 500, 1023

Zurbuchen, T. H., Fisk, L. A., Lepri, S. T., \& von Steiger, R. 2003, in AIP Conf. Proc. 679, Solar Wind Ten, ed. F. Malara et al. (Melville, NY: AIP), 604

Zurbuchen, T. H., \& Richardson, I. G. 2006, Space Sci. Rev., 123, 31 